\title{
Perioperative Management of Patients with Hemophilia during Spinal Surgery
}

\author{
Kazuyoshi Kobayashi, Shiro Imagama, Kei Ando, Kenyu Ito, Mikito Tsushima, Masayoshi Morozumi, \\ Satoshi Tanaka, Masaaki Machino, Kyotaro Ota, Yoshihiro Nishida, Naoki Ishiguro \\ Department of Orthopedic Surgery, Nagoya University Graduate School of Medicine, Nagoya, Japan
}

\begin{abstract}
Study Design: Single-center retrospective study.
Purpose: To optimize the perioperative management of patients with hemophilia who are undergoing spinal surgery.

Overview of Literature: Hemophilia is a rare disease in which there is a tendency of bleeding because of a congenital deficiency in blood coagulation factor activity. There has been no previous report on spinal surgery in patients with hemophilia.

Methods: The subjects were five patients (all males) with hemophilia who underwent spinal surgery at Nagoya University Hospital. Two patients had hemophilia A (deficiency of factor VIII) and three had hemophilia B (deficiency of factor IX). The mean age at the time of surgery was 63 years (range, 46-73 years). The following surgeries were performed: posterior lumbar interbody fusion (PLIF) in two patients, and lumbar fenestration, cervical laminoplasty and lumbar fenestration, and cervical laminoplasty and PLIF in one patient each.

Results: Coagulation factor at a mean dose of $4.8 \times 10^{3} \mathrm{U}\left(\right.$ range, $\left.3-6 \times 10^{3} \mathrm{U}\right)$ was intravenously injected before surgery, and a mean dose of $5.2 \times 10^{3} \mathrm{U}\left(\mathrm{rang}, 4-6 \times 10^{3} \mathrm{U}\right.$ ) was continuously administered for 24 hours after surgery. Factor activity was maintained at $\geq 80 \%$ until postoperative day 14 and at $\geq 50 \%$ thereafter. The average duration of surgery was 178 minutes (range, 133-233 minutes), the estimated blood loss was $661 \mathrm{~mL}$ (range, 272-1,344 mL), and a drain tube was left subfascially in place for 2 days in all patients. Reoperation due to postoperative surgical site infection was required in one patient, but there were no complications due to hemorrhagic diathesis. The total dose of coagulation factor administered during hospitalization was $102 \times 10^{3} \mathrm{U}$ (range, 46-198 $\times 10^{3} \mathrm{U}$ ). Conclusions: Coordination with a hematologist and dose adjustment of the coagulation factor preparation to maintain a target level of coagulation factor activity facilitated a smooth postoperative course with perioperative control of bleeding during spinal surgery for patients with hemophilia.
\end{abstract}

Keywords: Hemophilia A; Hemophilia B; Factor VIII; Factor IX; Blood loss; Spine

\section{Introduction}

Hemophilia is a rare disease that is characterized by a tendency of bleeding due to congenital deficiency of blood coagulation factor activity. Hemophilia A and B are the different types of the disease caused by the dysfunction of different coagulation factors. Patients with hemophilia may need surgical treatment for other diseases, and this requires consideration of pathologies caused by bleeding associated with hemophilia. The number of patients with

\footnotetext{
Received Aug 7, 2017; Revised Sep 11, 2017; Accepted Sep 11, 2017

Corresponding author: Shiro Imagama

Department of Orthopedic Surgery, Nagoya University Graduate School of Medicine, 65 Tsurumai Showa-ward, Nagoya, Aichi 4668550 , Japan

Tel: +81-52-833-6021, Fax: +81-52-833-6021, E-mail: imagama@med.nagoya-u.ac.jp
} 
hemophilia undergoing surgery is increasing annually, while bleeding problems in young patients are decreasing due to the development of hemostasis treatments. These factors have combined to improve life prognosis and increase the number of patients with hemophilia.

Surgical procedures inevitably result in bleeding, and excessive blood loss is particularly likely in spinal surgery for patients with hemophilia, which causes hesitancy in performing this surgery. There have been no reports on spinal surgery in patients with hemophilia. Here we report our experience of five such patients.

\section{Materials and Methods}

The subjects were five patients (all males) with hemophilia who underwent spinal surgery at Nagoya University Hospital. Two patients had hemophilia A (deficiency of factor VIII) and three had hemophilia B (deficiency of factor IX). Complications included one patient requiring dialysis and three patients with hepatitis $\mathrm{C}$. The mean age at the time of surgery was 63 years (range, 46-73 years). The following surgeries were performed: posterior lumbar interbody fusion (PLIF) in two patients, and lumbar fenestration, cervical laminoplasty and lumbar fenestration, and cervical laminoplasty and PLIF in one patient each. In all the patients, intraoperative hypotensive anesthesia was induced, and cotton fibrillar collagen (Integran; Nippon Zoki Pharmaceutical Co. Ltd., Osaka, Japan) was used for hemostasis. A closed suction drainage system was used in all surgeries. The mean preoperative activated partial thromboplastin time (APTT) was slightly prolonged to 43 seconds (range, 30-58 seconds), and the average preoperative coagulation factor activity was $21 \%$ (range, $3 \%-59 \%$ ). All factor allogeneic antibodies (inhibitors) were negative. Preoperative and postoperative outcomes and postoperative complications were examined. Preoperative coagulation factor activity was maintained at $\geq 80 \%$ preoperatively to postoperative day (POD) 14 , and at $\geq 50 \%$ after POD 15. An initial bolus administration of a single dose of the formulation was performed to obtain the target level, followed by continuous administration at $4-5 \mathrm{U} / \mathrm{kg} / \mathrm{hr}$, with consideration of the clearance rate of the factor preparation. This study was approved by the Ethics Committee of Nagoya University Hospital (IRB approval no., 354-3) and informed consent was obtained from all patients.

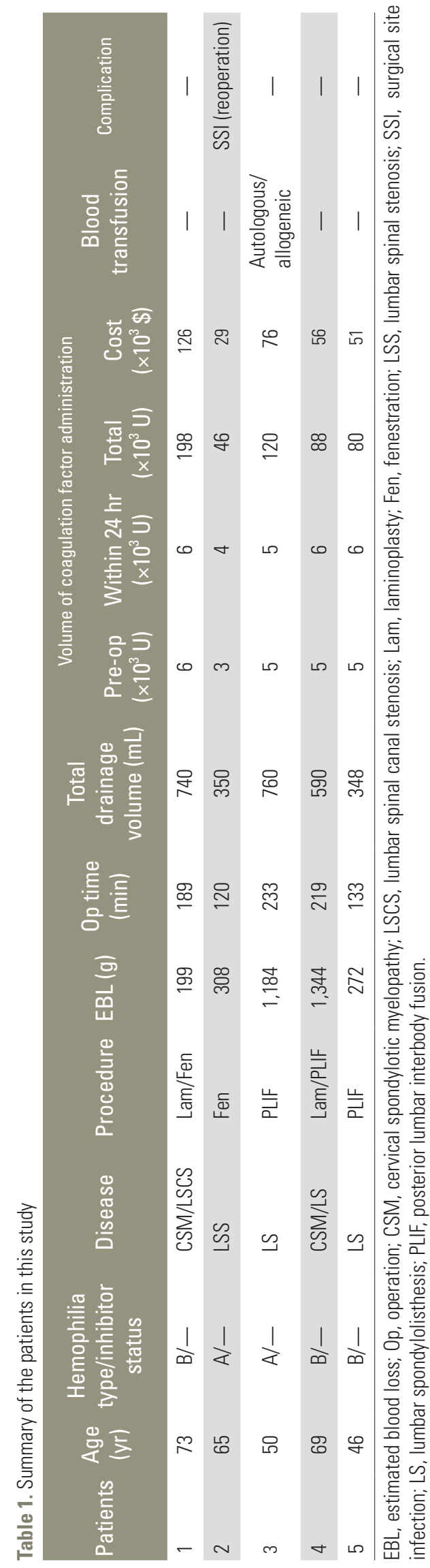




\section{Results}

We summarized five all cases in Table 1. Coagulation factor at a mean dose of $4.8 \times 10^{3} \mathrm{U}$ (range, $3-6 \times 10^{3} \mathrm{U}$ ) was intravenously injected just before surgery, followed by continuous administration of $5.2 \times 10^{3} \mathrm{U}$ (range, 4-6 $\times 10^{3} \mathrm{U}$ ) in the 24 hours after surgery. The factor activity was maintained at $\geq 80 \%$ until POD 14 , and at $\geq 50 \%$ thereafter. The average duration of surgery was $178 \mathrm{~min}$ utes (range, 133-233 minutes) and the estimated blood loss was $661 \mathrm{~mL}$ (range, 272-1,344 mL). The drain tube was left in place for 2 days, and the average total drainage volume for 2 days was $557 \mathrm{~mL}$ (range, 348-760 mL). Two patients received intraoperative blood salvage autotransfusion (IBSA). One patient required autologous and allogeneic blood transfusion (red cell concentrates mannitol adenine phosphate, 2 units). In one patient, reoperation was performed because of postoperative surgical site infection, but there were no complications due to hemorrhagic diathesis. The hospitalization period was 28 days (range, 19-40 days) and all patients were discharged. The total amount of coagulation factor administered during hospitalization was $102 \times 10^{3} \mathrm{U}$ (range, 46-198 $\times 10^{3} \mathrm{U}$ ). The average cost of perioperative blood coagulation factors was $\$ 65,000$ (range, $\$ 29,000-126,000$ ).

\section{Discussion}

Hemophilia is divided into hemophilia A and B, which are caused by qualitative and quantitative abnormalities of blood clotting factors VIII and IX, respectively. Hemophilia is a rare congenital recessive hereditary form of congenital hemorrhagic disease, with about 6,000 cases in Japan. Blood tests indicate prolongation of APTT, and characteristic clinical symptoms include deep bleeding, such as intraarticular and intramuscular bleeding. Advances in the development of blood products now enable factor activity to be maintained using coagulation factor preparations, and it is becoming possible to perform surgery in patients with hemophilia.

Administration of repeated doses of coagulation factor preparations over a prolonged period can lead to the appearance of inhibitors, with inhibitor generation occurring at the rate of $5 \%-10 \%$ in cases of repeated administration of factor preparations in patients with severe hemophilia [1]. An inhibitor will bind to the coagulation factor and cause functional impairment such that the hemostatic ef- fect induced using the supplemented coagulation factors markedly decreases or disappears. Our study did not include patients who exhibited inhibitor development, but it has been reported that massive bleeding can occur despite administration of a coagulation factor preparation due to the presence of inhibitors; thus, it is extremely important to confirm the absence of inhibitors before surgery [2].

A dosage regimen for the coagulation factor preparation should be established after confirming the pharmacokinetics in a test administration of the preparation before the surgery. Coagulation factor preparations may be given by bolus or continuous administration. Safe hemostatic management can be achieved with continuous administration without producing an unnecessarily high coagulation activity or a low trough [3]. The World Federation of Hemophilia Guidelines [4] recommend that an initial bolus administration of a single dose of the formulation is required to obtain the target level, followed by continuous administration at $4-5 \mathrm{U} / \mathrm{kg} / \mathrm{hr}$ considering the clearance rate of the factor preparation.

In spinal surgery, particular attention is required for intraoperative and perioperative bleeding management, including the risk of postoperative hematoma. In the patients of this study, controlled hypotensive anesthesia, cotton fibrillar collagen for hemostasis, and IBSA were used in combination to reduce intraoperative bleeding as much as possible, and this approach appeared to be effective. Intraoperative and postoperative bleeding were thus controlled with procedures used in normal spinal surgery.

There are reports suggesting that it is possible to reduce bleeding and blood transfusion volumes in spinal surgery by administering tranexamic acid during surgery $[5,6]$. For patients in whom allogeneic blood transfusion should be avoided, such as pediatric patients, it is important to prepare an adequate volume for autologous blood transfusion. Different techniques can be used for blood conservation, including proper positioning of the patient, muscle paralysis to minimize intra-abdominal pressure, IBSA, and use of antifibrinolytic agents [5,7-10]. In the postoperative management of patients with hemophilia, cases of massive bleeding have been reported outside the surgical site; thus, it is necessary to pay close attention to the risk of bleeding from non-wound sites [11,12]. Two of our patients with duration of surgery $>200$ minutes had intraoperative bleeding of $\geq 1,000 \mathrm{~mL}$. Therefore, surgeries of longer duration might lead to bleeding even when it is controlled with intravenous administration of coagula- 
tion factor, and careful bleeding management is required. However, in our study, controlled hypotensive anesthesia, autologous blood transfusion, and IBSA enabled management of intraoperative and postoperative bleeding.

In terms of medical costs, the use of perioperative blood coagulation factors costs an average of $\$ 65,000$, which is very expensive. Simultaneous surgeries on the cervical and lumbar spine were performed in two patients to try to reduce overall invasiveness and to shorten the duration of surgery. The high cost of blood coagulation factors is another reason to avoid multiple surgeries.

\section{Conclusions}

In our study, collaboration with a hematologist and an anesthesiologist, and dose adjustment of the coagulation factor preparation to maintain a target level of coagulation factor activity resulted in a good and smooth intraoperative and postoperative course with control of bleeding during and after spinal surgery in patients with hemophilia.

\section{Conflict of Interest}

No potential conflict of interest relevant to this article was reported.

\section{Acknowledgments}

Funding was from institutional sources only.

\section{References}

1. Shima M. Mechanism of inhibitor development and its management in patients with hemophilia. Jpn J Pediatr Hematol 1999;13:399-409.

2. Weiss AE. Circulating inhibitors in hemophilia A and B: epidemiology and methods of detection. In: Brinkhous KM, Hemker HC, editors. Handbook of hemophilia part II. Amsterdam: Excerpta Medica; 1975. p.629-46.
3. Takedani H, Kajiwara M. Continuous infusion with factor VIII concentrates during total joint arthroplasty for hemophilia A. Jpn J Thromb Hemost 2006;17:47-54.

4. World Federation of Hemophilia. Guidelines for the management of hemophilia [Internet]. Tokyo: Japan Blood Products Organization [cited 2017 Mar 20]. Available from: http://jbpo.or.jp/crossheart/pdf/ guideline.jp.

5. Elwatidy S, Jamjoom Z, Elgamal E, Zakaria A, Turkistani A, El-Dawlatly A. Efficacy and safety of prophylactic large dose of tranexamic acid in spine surgery: a prospective, randomized, double-blind, placebo-controlled study. Spine (Phila Pa 1976) 2008;33:2577-80.

6. Elgafy H, Bransford RJ, McGuire RA, Dettori JR, Fischer D. Blood loss in major spine surgery: are there effective measures to decrease massive hemorrhage in major spine fusion surgery? Spine (Phila Pa 1976) 2010;35(9 Suppl):S47-56.

7. Goodnough LT, Shander A, Brecher ME. Transfusion medicine: looking to the future. Lancet 2003;361:161-9.

8. Lawson JH, Murphy MP. Challenges for providing effective hemostasis in surgery and trauma. Semin Hematol 2004;41(1 Suppl 1):55-64.

9. Porte RJ, Leebeek FW. Pharmacological strategies to decrease transfusion requirements in patients undergoing surgery. Drugs 2002;62:2193-211.

10. Zheng F, Cammisa FP Jr, Sandhu HS, Girardi FP, Khan SN. Factors predicting hospital stay, operative time, blood loss, and transfusion in patients undergoing revision posterior lumbar spine decompression, fusion, and segmental instrumentation. Spine (Phila Pa 1976) 2002;27:818-24.

11. Yodono M. Anesthesia experience of hemophilia A caused postoperative transient consciousness. Rinsho-Masui 1979;3:1271-6.

12. Morimoto Y. Anesthesia experience of hepatectomy with hemophilia. Rinsho-Masui 1990;14:1027-8. 